\title{
The Influence of Hemispheric Solar Activity Features on the Interplanetary Plasma Parameters Throughout 1967-2017
}

\author{
M. A. El-Borie ${ }^{1}$, A. A. Thabet ${ }^{2}$, A. M. El-Taher ${ }^{3 *}$, H. MA. Zoheir ${ }^{1}$, and A. A. Bishara ${ }^{1}$ \\ ${ }^{(1)}$ Physics Department., Faculty of Science, Alexandria University, Alexandria, Egypt \\ ${ }^{(2)}$ Basic and Applied Science Department, Faculty of Engineering, Arab Academy for Science and Technology, Alexandria, Egypt \\ ${ }^{(3)}$ Physics and Chemistry Department, Faculty of Education, Alexandria University, Alexandria, Egypt
}

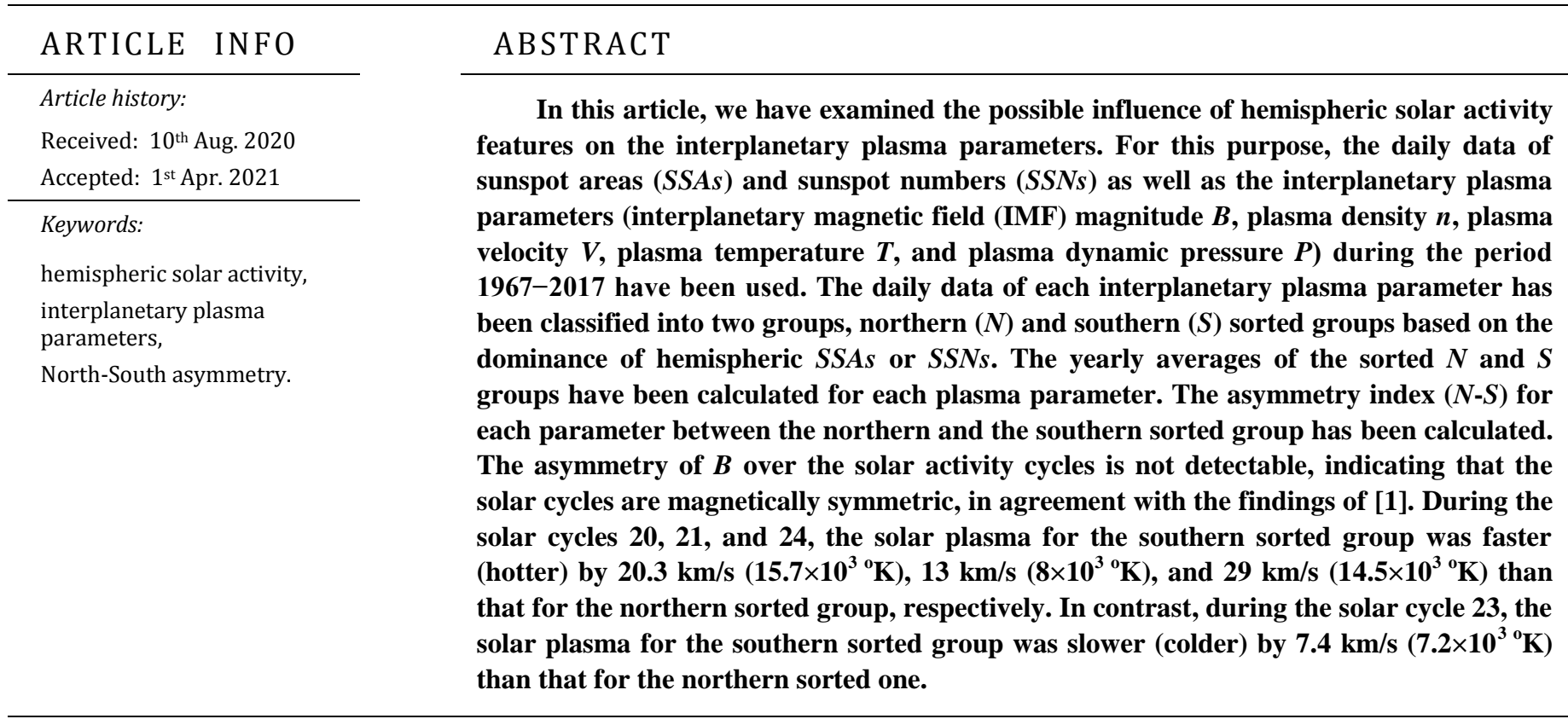

\section{INTRODUCTION}

It is well known for the solar physics community that the solar activity is the main structure of the Sun disk that is represented by the sunspot numbers ( $S S N s)$ and the sunspot areas (SSAs). The SSNs and SSAs are two criteria which have been used to characterize the long-term variations in the solar activity. D.H.Hathway [2] showed that SSNS and SSAs are indeed highly correlated $(r=0.994)$. In fact, a lead or lag between these two quantities during each solar cycle is not confirmed. With the exception of one thing, the zero point, the two quantities could almost be used interchangeably. There is a slight shift from a zero point of SSAs from those SSNs. Therefore, Li et al. [3] introduced a new parameter (an indicator), the sunspot unit areas (SUAs). This parameter is defined as the ratio between $S S A$ to $S S N$, i.e., it describes the average size of sunspots produced by the dynamo in a solar cycle. Significantly, the durations of solar activity varied in the northern and southern solar hemispheres. Various features of solar activity reveal non-uniformity on the solar disk. As a result, it has been found that, in one of the solar hemispheres (northern or southern) more phenomena occur simultaneously than in other. This phenomenon, with respect to the solar equator, is regarded as the North-South $(N-S)$ asymmetry. The $N-S$ asymmetries are considered as statistically significant in solar activity [4]. In addition, it has been observed that some common features of $N-S$ asymmetry are present while studying different forms of solar activity, e.g. sunspots, solar flares, or sudden disappearances of solar prominences [5]. For example, $78 \%$ of solar flares were detected in the northern hemisphere during the dominance time of northern sunspot [6]. The main period in the $N-S$ asymmetry of the sunspot groups is about 80 years [7] as well as, in the interplanetary space, the $\mathrm{N}-\mathrm{S}$ asymmetry is essentialyl affecting both interplanetary and near Earth- 
space parameters. For example, this influences the position of the heliospheric current sheet (HCS) and results in a difference in the number of toward and away interplanetary magnetic field (IMF) sector days [8- 10]. Regarding the heliographic equator, the asymmetrical distribution of the solar wind has been identified [11, 12]. Moreover, the $N-S$ asymmetry of interplanetary magnetic field magnitude $(B)$ has been examined [13, 14]. Furthermore, Makarov [15] studied the $N-S$ asymmetry of geomagnetic activity and solar wind electric field according to the sign of the IMF sector structure from (to) the Sun which represents positive (negative) sector. The results revealed that in the positive IMF sector, the level of geomagnetic activity in the northern hemisphere was higher than that in the southern one, whereas in the negative sector it was higher in the southern hemisphere. In addition, it was established that the higher the magnetic activity, the greater wasthe hemispheric asymmetry and during quiet periods, the asymmetry was not present.

Recently, the solar and plasma parameters have been investigated based on the IMF polarity sectors [1]. Various parameters have been examined. The dependence of IMF magnitude, $B$ on the solar activity cycle does not exist. The solar plasma was faster by $\sim 13$ $\mathrm{km} / \mathrm{s}$ and $\sim 16 \mathrm{~km} / \mathrm{s}$ for toward polarity days than that of days of away polarity during the solar cycles 21 and 22, respectively. In comparison, the solar plasma was slower (by $\sim 17.5 \mathrm{~km} / \mathrm{s}$ ) for toward polarity days than those of away polarity during the solar cycle 23 . Furthermore, during the solar cycles 21,22 , and 24, the solar plasma was hotter for days of toward polarity than those of away polarity, whereas during the solar cycles 20 and 23, the solar plasma was cooler for days of toward polarity than those of away polarity. On the other hand, the geomagnetic activity indices ( $a a, K p, A p$, and $D s t$ ) have been examined on the basis of the asymmetry between the directions of the IMF above and below of the HCS [16]. The study confirmed the existence of significant yearly $\mathrm{N}-\mathrm{S}$ asymmetries of geomagnetic indices observed throughout the entire period (1967-2016). Whereas, throughout the epoch of positive magnetic polarity (1971-1979), the geomagnetic indices ( $a a, A p$ and $K p)$ exhibited northern dominances. In contrast, during the negative magnetic polarity epoch $(2001-2012)$, the asymmetries shifted to the southern solar hemisphere. More recently, El-Borie et al. [17] studied the influence of hemispheric distribution of solar activity feature represented by SSAs on the geomagnetic indices during the period 1967-2016. The results confirmed the possible connection between geomagnetic activity disturbances and the sign of the dominance of the solar activity, i.e. the $N-S$ asymmetry of geomagnetic activity. The geomagnetic disturbances have also revealed major asymmetrical states in different periods. In addition, the geomagnetic activity has southern dominance during solar cycle 22 whereas it shifted to the northern activity during solar cycle 23. The dependency of the geomagnetic activity disturbance of $a a, A p, K p$, and $D s t$ indices over the solar activity cycle is verified and the sign of the asymmetry shifts from one solar cycle to another.

The present study aims to investigate the influence of solar activities ( $S S A s$ and $S S N S$ ) on the interplanetary plasma parameters i.e., the N-S asymmetry of the IMF magnitude $(B)$, the plasma density $n$, the plasma temperature $T$, the dynamic pressure $P$, and plasma velocity $V$ based on both the dominance of hemispheric distributions of SSAs and SSNS on the solar disc throughout the period 1967-2017 (which covers nearly five solar activity cycles 20-24).

\section{Observational Data}

2.1 Daily averages of solar plasma velocity $(V)$, plasma temperature $(T)$, plasma density $(n)$, plasma dynamic pressure $(P)$, and IMF magnitude $(B)$ from Jan 1967 to Dec 2017 were downloaded via http://omniweb.gsfc.noaa.gov/from/dx1.html.

2.2 Daily hemispheric SSAs (northern and southern) from 1967 to 2017 were taken from Royal Greenwich Observatory and USAF/NOAA dataset.

2.3 Daily hemispheric SSNs from Jan 1992 to Dec 2017 were downloaded from (http://www.sidc.be./silso/infosndhem).

\section{RESULTS AND DISCUSSION}

\subsection{The N-S Asymmetry for The Interplanetary Plasma Parameters According to Hemispheric Distribution of SSAs}

In this section, we have used the daily averages of IMF magnitude $(B)$ and solar-plasma parameters (solar plasma velocity $V$, plasma density $n$, plasma temperature $T$, and plasma dynamic pressure $P$ ), taken near 1 AU by a variety of spacecraft over the time interval 1967-2017. These interplanetary plasma parameters have been analyzed according to the dominance of hemispheric solar activity indices (SSAs and SSNS) to examine the possible presence of $N-S$ asymmetry. In order to study the relationship between the solar wind parameters and the solar activity indices (SSNs and SSAs), the previous studies of $[16,18]$ indicated that a time-lag $(2-y r$ for $T$, $V$, and $P$ as well as 1 -yr for $n$ ) should be taken into 
account as well as the data of the interplanetary plasma parameters and the sunspot indices need to be smoothed over 3 years. Data of the daily averages of the interplanetary plasma parameters has been classified into two groups, namely northern $(N)$ and southern $(S)$ groups based on the dominance (sign) of the solar activity in the solar hemisphere of that day. The yearly average has been calculated for each group $(N$ and $S$ ) for each considered plasma parameter. Finally, the $N-S$ asymmetry index is calculated for each parameter. As a result, if $(N-S)>0$, the parameter value in $N$ group dominates of that in the $S$ group, otherwise, the reverse is true.
To reveal, in detail, the influence of the dominanace of SSAs in the northrern and southern hemispheres on the selected interplanetry plasma parameters, Figures (1 to 3 ) display the $N-S$ asymmetries of $B, n, V, T$, and $P$, respectively. The vertical dashed lines indicate the times of solar activity minimum. The horizontal dashed lines up and down the zero level indicate the $95 \%$ confidence level for positive and negative values, respectively. For more accurate results, only the points nearer to or above the $95 \%$ confidence level are considered as significant in the following Figures.
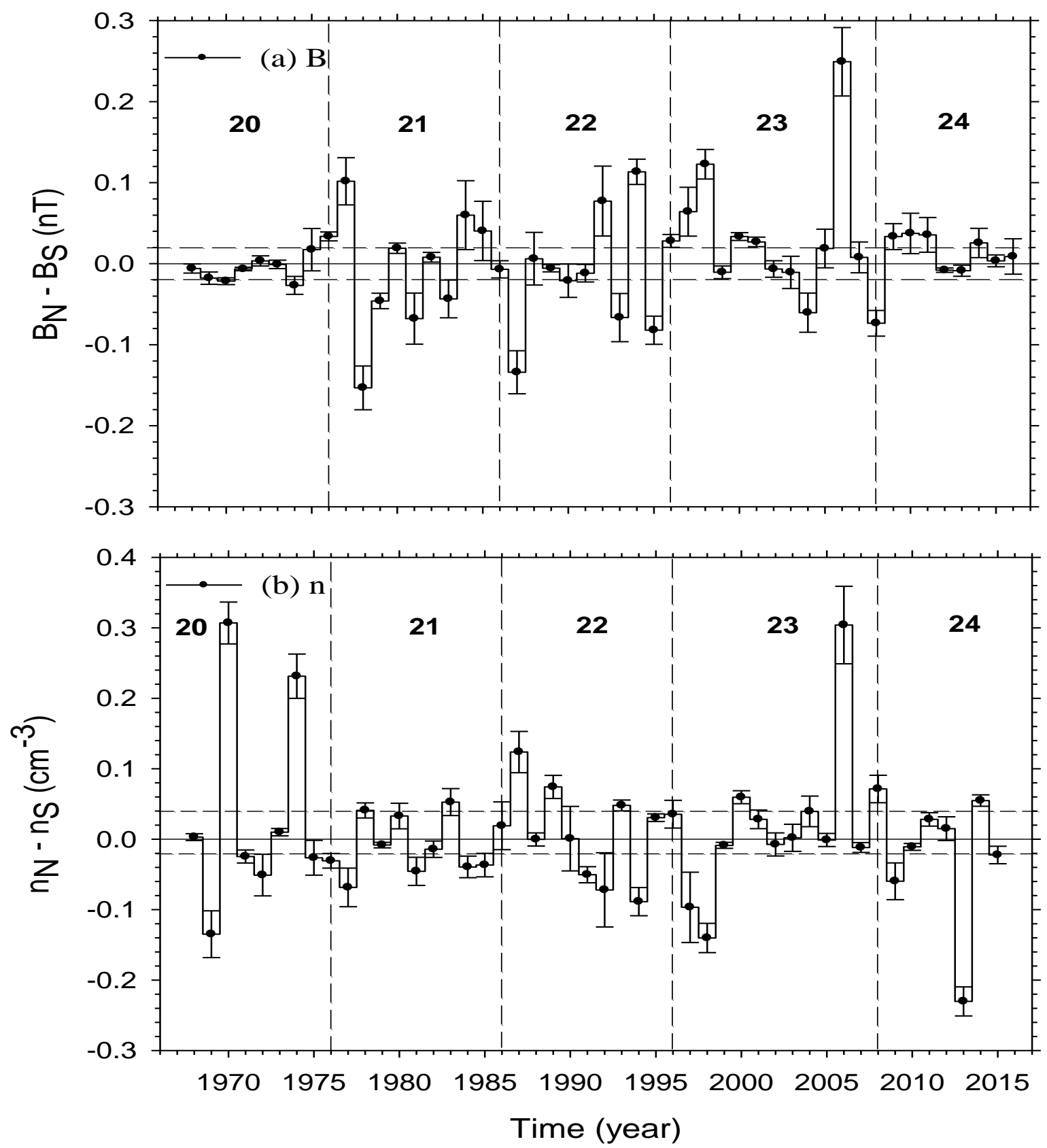

Fig (1): The 3-yr running averages of N-S asymmetries for IMF magnitude (plot a) and for plasma density (plot b) over the period (1967-2017). The vertical dashed lines denote the minima of solar activity. The horizontal dashed lines represent the 95\% confidence levels for positive and negative values of N-S asymmetry. The solar cycles are numbered on each plot 
Figure 1a represents the $N-S$ asymmetry between the northern and the southern groups of $B$ during the entire period (1967-2017). Plot (1a) of $B$ displays that there are eight years of dominance of northern group which occurred through the solar cycle 20 (in 1976), cycle 21 (1977), cycle 22 (1992 and 1994), cycle 23 (1997-1998, 2000, and 2006). The most prominent peak of northern sorted group is located at $2006(0.25 \pm 0.042$ $\mathrm{nT}$ ) during the descending phase of cycle 23 . On the other hand, within the $95 \%$ confidence level, one can observe eight years dominance of the sorted southern group throughout the cycles 20-23. These southern sorted group years are distributed as follow; three years occurred in cycle 21 (1978, 1979 and 1981), three years in cycle 22 (1987, 1993, and 1995), and two years in cycle 23 (in 2004 and 2008). The highest significant peaks for southern sorted group occurred in 1978 $(0.15 \pm 0.027 \mathrm{nT})$ and $1987(0.134 \pm 0.027 \mathrm{nT})$ during the ascending phases of cycles 21 and 22, respectively.

Figure (1) plot (b) presents the asymmetry between the northern and the southern sorted groups for ion plasma density $(n)$. It is noted that there are eight years in which the plasma was denser for the northern sorted group than that for the southern sorted one. They are distributed in the solar cycles as follows; two in cycle 20 (1970 and 1974), two in cycle 22 (1987 and 1989), three in cycle 23 (2000, 2006, and 2008), and one in cycle 24 (2014). In contrast, within the limit of accuracy (the $95 \%$ confidence level), the plot demonstrates eight years in which the plasma for the southern sorted group was denser than that of the northern sorted one. One year having dominance of the southern group is located in cycle 20 (1969), one in cycle 21 (1977), two in cycle 22 (1991 and 1994), two in cycle 23 (1997 and 1998), and two in cycle 24 (2009 and 2013). The highly northern significant peaks (in a descending order) are found in $1970\left(0.306 \pm 0.029 \mathrm{~cm}^{-3}\right), 2006\left(0.303 \pm 0.055 \mathrm{~cm}^{-3}\right)$, and $1974\left(0.231 \pm 0.031 \mathrm{~cm}^{-3}\right)$. On the other hand, the most prominent peak for the is located in $2013\left(0.230 \pm 0.031 \mathrm{~cm}^{-3}\right)$.

Figure (2a) presents the asymmetry between the northern and the southern sorted groups for the solar plasma velocity $(V)$ during the entire period. Within the limit of accuracy (95\% confidence level), there are six and ten years, that significant positive and negative asymmetries, respectively. For the northern sorted group, the solar plasma was faster than those of the southern sorted one in ten years during the solar cycles 20-23. One northern asymmetry occurred during solar cycle 20 (in 1970), one in cycle 21 (in 1986), one in cycle 22 (in 1994), and three prominent ones in cycle 23 (2000, 2006 and 2008). On the other hand, for the southern sorted group, the solar plasma was faster than that of the northern sorted one for fourteen years during the considered solar cycles. Two asymmetries (in 1971 and 1974) were observed in cycle 20, four (1977- 1978,
1983, and 1985) in cycle 21, two (1993 and 1996) in cycle 22, and finally two (2011 and 2013) in cycle 24 . Moreover, the solar plasma for northern sorted group was faster by $12.4 \pm 1.3 \mathrm{~km} / \mathrm{s}$ (in 1970) and $12.2 \pm 1.8 \mathrm{~km} / \mathrm{s}$ (in 2000) than that of the southern sorted group. Regarding the southern sorted group, the solar plasma was faster by $7 \pm 1.8 \mathrm{~km} / \mathrm{s}$ (in 1971) and $7.9 \pm 0.86 \mathrm{~km} / \mathrm{s}$ (in 2013) than that of the northern sorted group.

Figure (2b) displays the asymmetry between the northern and the southern sorted groups for plasma temperature $(T)$ during the considered period. As there is a direct proportionality between the plasma kinetic energy and its absolute temperature, one can note that both $N-S$ asymmetry plots of $V$ and $T$ exhibit coupling at some level (both plots showed nearly symmetric behavior). Seven random years showed that the solar plasma was hotter for the northern sorted group than the southern sorted one. The locations of $N-S$ asymmetries of $T$ are as follow; one (1970) in cycle 20, one (1986) in cycle 21, two (1994 and 1995) in cycle 22, and three (2000, 2006, and 2008) in cycle 23. In contrast, in nine out of 47 years, the solar plasma was hotter in the southern sorted group than the northern sorted one. Two of these asymmetries located in cycle 20 (1971 and 1974), three in cycle 21 (1977, 1983, and 1985), two (1993 and 1996) in cycle 22, and two (2011 and 2013) in cycle 24 . Regarding the northern sorted group, the solar plasma was hotter than those of southern group by $9.08 \times 10^{3} \pm 0.94 \times 10^{3}{ }^{\mathrm{o}} \mathrm{K}, \quad 6.67 \times 10^{3} \pm 0.96 \times 10^{3}{ }^{\mathrm{o}} \mathrm{K}$, and $7.1 \pm 1.23{ }^{\circ} \mathrm{K}$ in 1970 (during the ascending phase of cycle 20), 2000, and 2008 (at minimum of solar activity), respectively. On the other hand, regarding the southern sorted group, the solar plasma was hotter by $5.27 \times 10^{3} \pm 1.30 \times 10^{3}{ }^{\mathrm{o}} \mathrm{K}$ and by $4.63 \times 10^{3} \pm 0.43 \times 10^{3}{ }^{\mathrm{o}} \mathrm{K}$ in 1971 (during the ascending phase of cycle 20) and in 2013 (near to maximum activity of solar cycle 24).

The asymmetry of plasma dynamic pressure $(P)$ between the northern and the southern sorted groups is displayed in Figure (3). Within the limit of accuracy, one can observe ten years in which the plasma dynamic pressure for the northern sorted group predominated that of the southern sorted one. These significant asymmetries occurred at cycle 20 (1970), cycle 21 (1980 and 1986), cycle 22 (1989, 1991, 1994, and 1996), and cycle 23 (2000, 2006, and 2008). On the other hand, twelve years in which the plasma dynamic pressure for southern sorted group predominated that of the northern sorted one. Four of these negative asymmetries occurred in cycle 20 (1968, 1971, and 1976), three in cycle 21 (1977, 1978 and 1983), two in cycle 22 (1987 and 1993), one in cycle 23 (in 2004), and three in cycle 24 (2011, 2013, and 2014). The most significant northern and southern asymmetries are $0.117 \pm 0.012 \mathrm{nPa}$ and $0.075 \pm 0.0041 \mathrm{nPa}$ occurred in 1970 and 2013, respectively. 


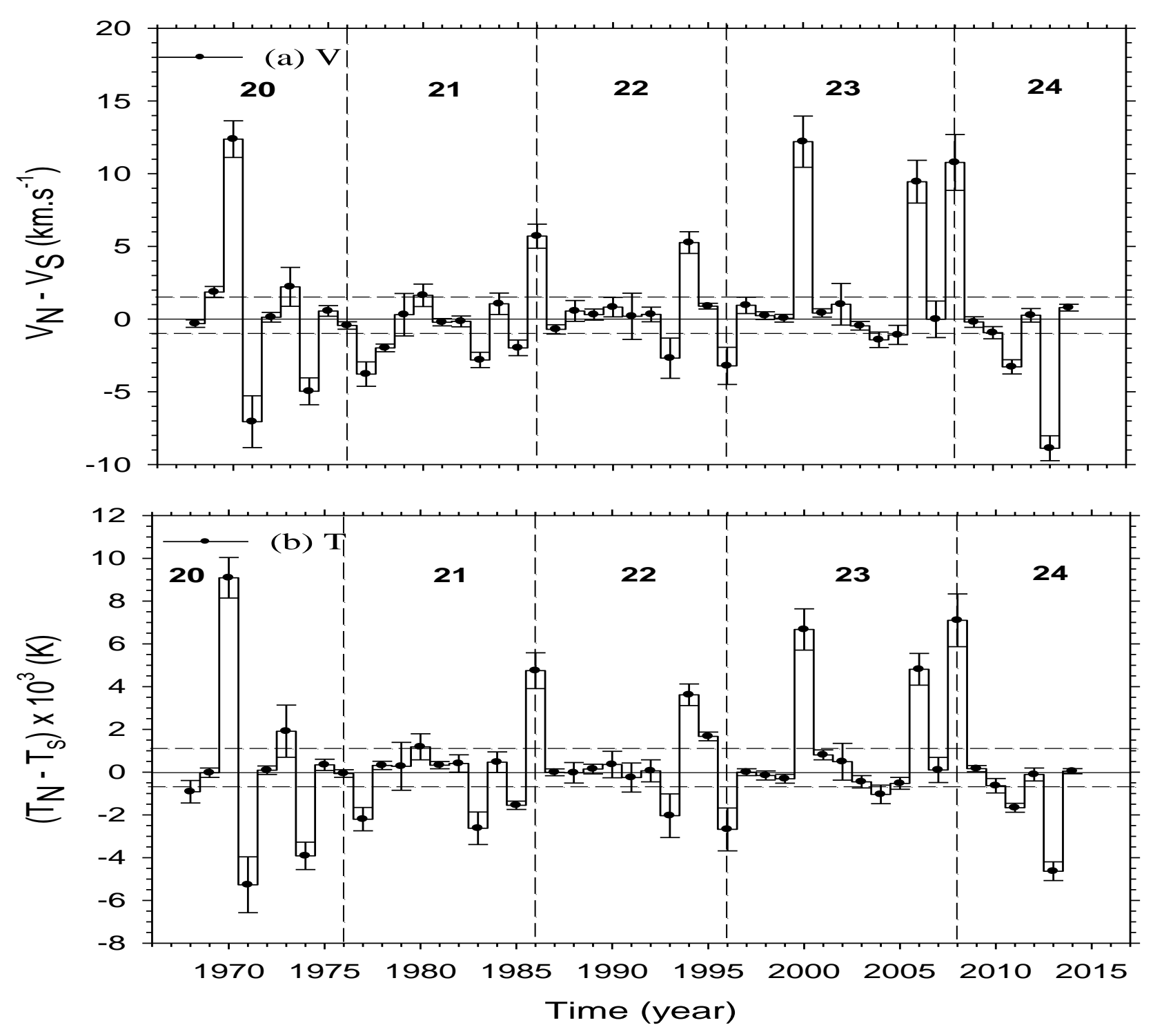

Fig. (2): The 3-yr running averages of N-S asymmetries for solar plasma velocity (plot a) and for plasma temperature (plot b) over the period (1967-2017). The notations are the same as in Fig. (1)

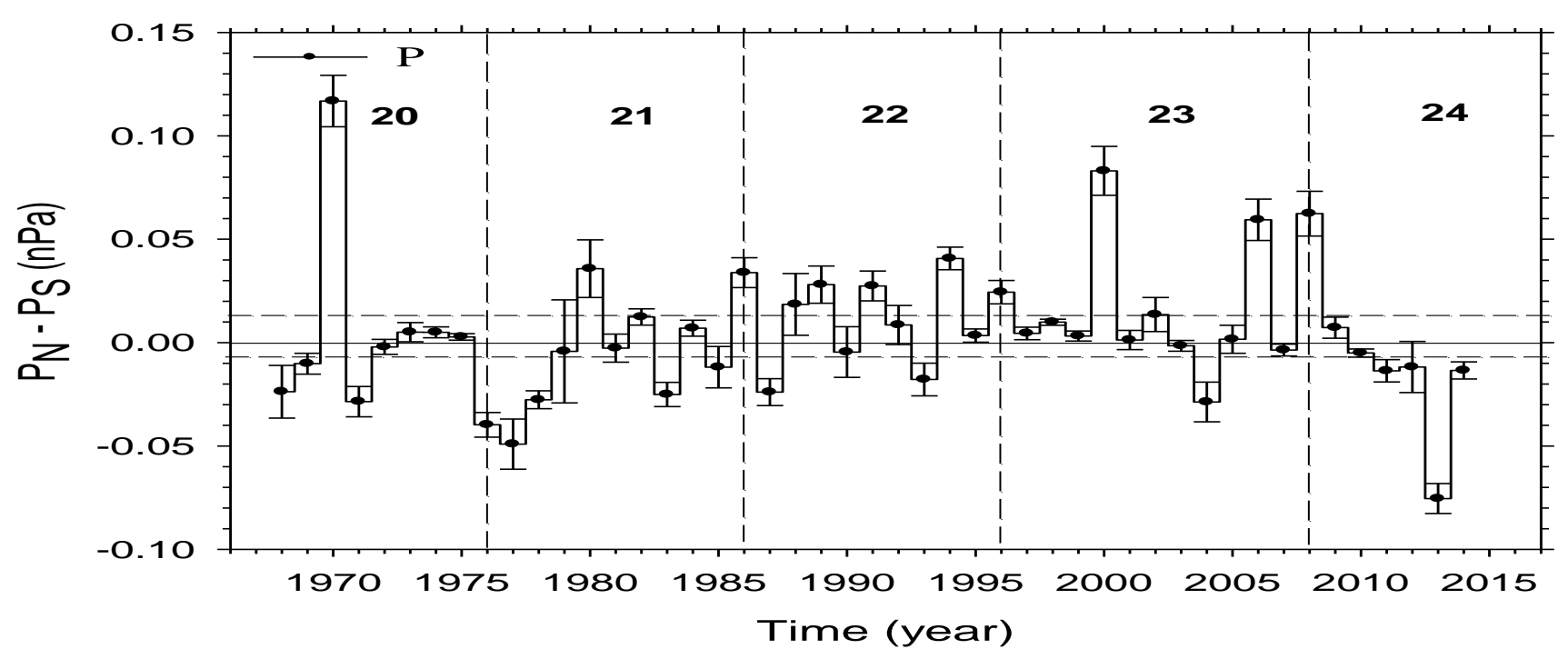

Fig. (3): The 3-yr running averages of N-S asymmetries for solar plasma pressure (plot a) over the period (1967-2017). The notations are the same as in Fig. (1) 
Table (1): Grand averages of IMF magnitude, solar and plasma parameters during solar activity cycle 20 based on the hemispheric SSAs. The number of the northern and the southern days and their percentages are also listed. Note that, the solar cycle 20 started in Oct. 1964 whereas the measurements were not available before Jan. 1967.

\begin{tabular}{cccc}
\hline $\begin{array}{l}\text { IMF magnitude, } \\
\text { solar, and plasma } \\
\text { parameters }\end{array}$ & $\begin{array}{l}\text { No. northern days } \\
\mathbf{1 6 7 8}\end{array}$ & $\begin{array}{c}(\%) \\
\mathbf{5 3 . 2}\end{array}$ & \multicolumn{3}{c}{ No. southern days (\%) } \\
$\mathbf{1 4 7 8}$ & $\mathbf{4 6 . 8}$ & \\
\hline & Average & Average & $(\mathrm{N}-\mathrm{S})$ \\
$\mathrm{SSAs}(\mu \mathrm{Hemi})$ & $557 \pm 11.48$ & $434 \pm 8.81$ & 123 \\
$\mathrm{~B}(\mathrm{nT})$ & $6.24 \pm 0.06$ & $6.29 \pm 0.07$ & -0.05 \\
$\mathrm{n}\left(\mathrm{cm}^{-3}\right)$ & $6.36 \pm 0.14$ & $6.69 \pm 0.13$ & -0.33 \\
$\mathrm{~T} \times 10^{3}\left({ }^{\mathrm{o}} \mathrm{K}\right)$ & $125.3 \pm 2.80$ & $141 \pm 2.86$ & -15.7 \\
$\mathrm{~V}(\mathrm{~km} / \mathrm{s})$ & $443.7 \pm 2.75$ & $464 \pm 3.12$ & -20.3 \\
$\mathrm{P}(\mathrm{nPa})$ & $2.50 \pm 0.04$ & $2.64 \pm 0.04$ & -0.14 \\
\hline
\end{tabular}

Table (2): Grand averages of IMF magnitude, solar and plasma parameters during solar activity cycle 21 based on the hemispheric SSAs. The number of the northern and the southern days and their percentages are also listed.

\begin{tabular}{cccc}
\hline $\begin{array}{l}\text { IMF magnitude, } \\
\text { solar, and plasma } \\
\text { parameters }\end{array}$ & $\begin{array}{c}\text { No. northern days } \\
\mathbf{1 7 0 1}\end{array}$ & $\begin{array}{c}(\%) \\
\mathbf{4 4 . 3}\end{array}$ & \multicolumn{3}{c}{ No. southern days (\%) } \\
$\mathbf{1 7 5 2}$ & $\mathbf{4 5 . 7}$ & \\
\hline & Average & Average & $(\mathrm{N}-\mathrm{S})$ \\
$\mathrm{SSAs}(\mu \mathrm{Hemi})$ & $594 \pm 12$ & $613 \pm 13$ & -19 \\
$\mathrm{~B}(\mathrm{nT})$ & $7.23 \pm 0.070$ & $7.27 \pm 0.075$ & -0.04 \\
$\mathrm{n}\left(\mathrm{cm}^{-3}\right)$ & $8.00 \pm 0.13$ & $7.78 \pm 0.130$ & 0.22 \\
$\mathrm{~T} \times 10^{3}\left({ }^{\mathrm{o}} \mathrm{K}\right)$ & $112 \pm 2.25$ & $120 \pm 2.49$ & -8 \\
$\mathrm{~V}(\mathrm{~km} / \mathrm{s})$ & $433 \pm 2.54$ & $446 \pm 2.67$ & -13 \\
$\mathrm{P}(\mathrm{nPa})$ & $2.68 \pm 0.05$ & $2.87 \pm 0.05$ & -0.19 \\
\hline
\end{tabular}

Table (3): Grand averages of IMF magnitude, solar and plasma parameters during solar activity cycle 22 based on the hemispheric SSAs. The number of the northern and the southern days and their percentages are also listed.

\begin{tabular}{ccccc}
\hline $\begin{array}{l}\text { IMF magnitude, } \\
\text { solar, and plasma } \\
\text { parameters }\end{array}$ & $\begin{array}{l}\text { No. northern days } \\
\mathbf{1 5 8 1}\end{array}$ & $\begin{array}{c}\text { (\%) } \\
\mathbf{4 3 . 6}\end{array}$ & \multicolumn{3}{c}{ No. southern days (\%) } & \\
\hline & Average & Average & $(\mathrm{N}-\mathrm{S})$ \\
$\mathrm{SSAs}(\mu \mathrm{Hemi})$ & $540 \pm 12.8$ & $614 \pm 14$ & -74 \\
$\mathrm{~B}(\mathrm{nT})$ & $7.04 \pm 0.09$ & $7.26 \pm 0.094$ & -0.22 \\
$\mathrm{n}\left(\mathrm{cm}^{-3}\right)$ & $8.35 \pm 0.15$ & $8.12 \pm 0.154$ & 0.23 \\
$\mathrm{~T} \times 10^{3}\left({ }^{\mathrm{o}} \mathrm{K}\right)$ & $111.2 \pm 3.26$ & $111.7 \pm 2.49$ & -0.5 \\
$\mathrm{~V}(\mathrm{~km} / \mathrm{s})$ & $437 \pm 2.7808$ & $442 \pm 2.968$ & -5 \\
$\mathrm{P}(\mathrm{nPa})$ & $2.86 \pm 0.05$ & $2.86 \pm 0.047$ & 0 \\
\hline
\end{tabular}

Table (4): Grand averages of IMF magnitude, solar and plasma parameters during solar activity cycle 23 based on the hemispheric SSAs. The number of the northern and the southern days and their percentages are also listed.

\begin{tabular}{cccc}
\hline $\begin{array}{l}\text { IMF magnitude, } \\
\text { solar, and plasma } \\
\text { parameters }\end{array}$ & $\begin{array}{l}\text { No. northern days } \\
\mathbf{1 6 0 1}\end{array}$ & $\begin{array}{c}(\%) \\
\mathbf{3 5 . 3}\end{array}$ & \multicolumn{3}{c}{ No. southern days (\%) } & \\
$\mathbf{2 2 1 9}$ & $\mathbf{4 8 . 9}$ & \\
\hline & Average & Average & $(\mathrm{N}-\mathrm{S})$ \\
$\mathrm{SSAs}(\mu \mathrm{Hemi})$ & $377 \pm 8.01$ & $434 \pm 8.12$ & -57 \\
$\mathrm{~B}(\mathrm{nT})$ & $6.76 \pm 0.074$ & $6.31 \pm 0.057$ & 0.45 \\
$\mathrm{n}\left(\mathrm{cm}^{-3}\right)$ & $6.25 \pm 0.10$ & $6.00 \pm 0.081$ & 0.25 \\
$\mathrm{~T} \times 10^{3}\left({ }^{\mathrm{o}} \mathrm{K}\right)$ & $109.4 \pm 1.99$ & $102.2 \pm 1.70$ & 7.2 \\
$\mathrm{~V}(\mathrm{~km} / \mathrm{s})$ & $451.8 \pm 2.54$ & $444.4 \pm 2.3$ & 7.4 \\
$\mathrm{P}(\mathrm{nPa})$ & $2.19 \pm 0.035$ & $2.05 \pm 0.03$ & 0.14 \\
\hline
\end{tabular}

Table (5): Grand averages of IMF magnitude, solar and plasma parameters during solar activity cycle 24 based on the hemispheric SSAs. The number of the northern and the southern days and their percentages are also listed.

\begin{tabular}{cccc}
\hline $\begin{array}{l}\text { IMF magnitude, } \\
\text { solar, and plasma } \\
\text { parameters }\end{array}$ & $\begin{array}{l}\text { No. northern days } \\
\mathbf{1 5 7 4}\end{array}$ & $\begin{array}{c}(\%) \\
\mathbf{4 7 . 9}\end{array}$ & \multicolumn{3}{l}{ No. southern days (\%) } \\
$\mathbf{1 2 0 6}$ & $\mathbf{3 6 . 7}$ & \\
\hline & Average & Average & $(\mathrm{N}-\mathrm{S})$ \\
$\mathrm{SSAs}(\mu \mathrm{Hemi})$ & $274 \pm 5.94$ & $288 \pm 8.12$ & -14 \\
$\mathrm{~B}(\mathrm{nT})$ & $5.46 \pm 0.057$ & $5.78 \pm 0.069$ & -0.32 \\
$\mathrm{n}\left(\mathrm{cm}^{-3}\right)$ & $6.01 \pm 0.11$ & $6.44 \pm 0.12$ & -0.43 \\
$\mathrm{~T} \times 10^{3}\left({ }^{\mathrm{o}} \mathrm{K}\right)$ & $82.40 \pm 1.87$ & $96.92 \pm 2.14$ & -14.52 \\
$\mathrm{~V}(\mathrm{~km} / \mathrm{s})$ & $409 \pm 2.57$ & $438 \pm 2.92$ & -29 \\
$\mathrm{P}(\mathrm{nPa})$ & $1.91 \pm 0.033$ & $2.16 \pm 0.038$ & 0.25 \\
\hline
\end{tabular}

Tables (1-5) present the grand averages of $S S A_{S}, B$, $n, T, V$, and $P$ for the northern and the southern sorted groups over the solar cycles 20-24. Also, the number of the northern and the southern days and their percentage with respect to the total number of days during each solar cycle are addressed. According to the SSAs, the solar cycle 20 exhibited the predominance of the northern hemispheric activity over the southern one by $12.4 \%$. On the other hand, the southern hemispheric activity predominates the northern one by $\sim 6 \%$ and $7 \%$ in solar cycles 22 and 23, respectively. Furthermore, during the solar cycles 21 and 24, the solar activity exhibited nearly symmetrical distribution in both solar hemispheres. Additionally, the asymmetry between the northern and the southern groups for the grand averages of $B$ and $n$ over the solar activity cycles is undetectable. On other word, the asymmetry of the grand average of $B$ based on the solar cycle generally is not noticeable. One can conclude that, the solar cycles are magnetically symmetric. Previous study of non-uniformity of solar and plasma parameters on the basis of the IMF polarities (toward and away) revealed that the grand average of IMF magnitude over the solar cycle (20-24) was not present [1]. Also, the solar plasma for the southern sorted group was hotter by $15.7 \times 10^{3}{ }^{\circ} \mathrm{K}, 8 \times 10^{3}{ }^{\circ} \mathrm{K}$ and $14.5 \times 10^{3}{ }^{\circ} \mathrm{K}$ than that for the northern sorted group during the solar cycles 20,21 , and 24 , respectively. In contrast, during the solar cycle 23 , the solar plasma for the southern sorted group was colder by $7.2 \times 10^{3}{ }^{\circ} \mathrm{K}$ than that for the northern sorted group. Moreover, the solar plasma for the southern sorted group was faster by 20.3 $\mathrm{km} / \mathrm{s}, 13 \mathrm{~km} / \mathrm{s}, 5 \mathrm{~km} / \mathrm{s}$, and $29 \mathrm{~km} / \mathrm{s}$ than that for the northern sorted group during the solar cycles 20, 21, 22, and 24 , respectively. In contrast, during the solar cycle 23 , the solar plasma for the southern sorted group was slower by $7.4 \mathrm{~km} / \mathrm{s}$ than that for the northern sorted one. 
3.3. The N-S asymmetry for the interplanetary plasma parameters according to the dominance of hemispheric $\mathrm{SSNs}$

Daily averages of IMF magnitude $(B)$ and interplanetary plasma parameters $(n, V, T$, and $P$ ) over the period 1992-2017, have been investigated according to the dominance of hemispheric SSNs. As has been done in section 3.2, the daily data of $B, n, V, T$, and $P$ has been classified into two groups; Northern and Southern on the basis of the dominance of hemispheric distribution of SSNS in that day. To investigate the influence of the solar activity indices (SSNs and SSAs) on the solar wind parameters, a time-lag should be taken into our consideration as well as the data of both solar activity indices and solar wind parameters should be smoothed over a window of 3 -yr. The yearly average has been calculated for each hemispheric group $(N$ and $S)$ for each considered plasma parameter. Finally, the $\mathrm{N}-\mathrm{S}$ asymmetry index is calculated for each parameter.

Figure (4) displays the N-S for the IMF magnitude $B$ (top plot) and plasma density $n$ (bottom plot). Vertical midpoint steps indicate the centered 3-year running averages over the period 1992-2017. Estimated error is displayed for each yearly average value for each parameter. The top plot, $\left[\mathrm{B}_{\mathrm{N}}-\mathrm{B}_{\mathrm{S}}\right]$, demonstrates four positive N-S asymmetries occurring in 1994, 1997, 1998, and 2006. In contrast, the negative N-S asymmetries are revealed through 1993, 1995, 2004, and 2008. The highest significant asymmetry with a northern tendency is $(0.232 \pm 0.038 \mathrm{nT})$ occurring in 2006 (through the solar cycle 23) while the most prominent southern asymmetry is $(-0.115 \pm 0.016 \mathrm{nT}$ ) occurring in 1995 (through the solar cycle 22 ).
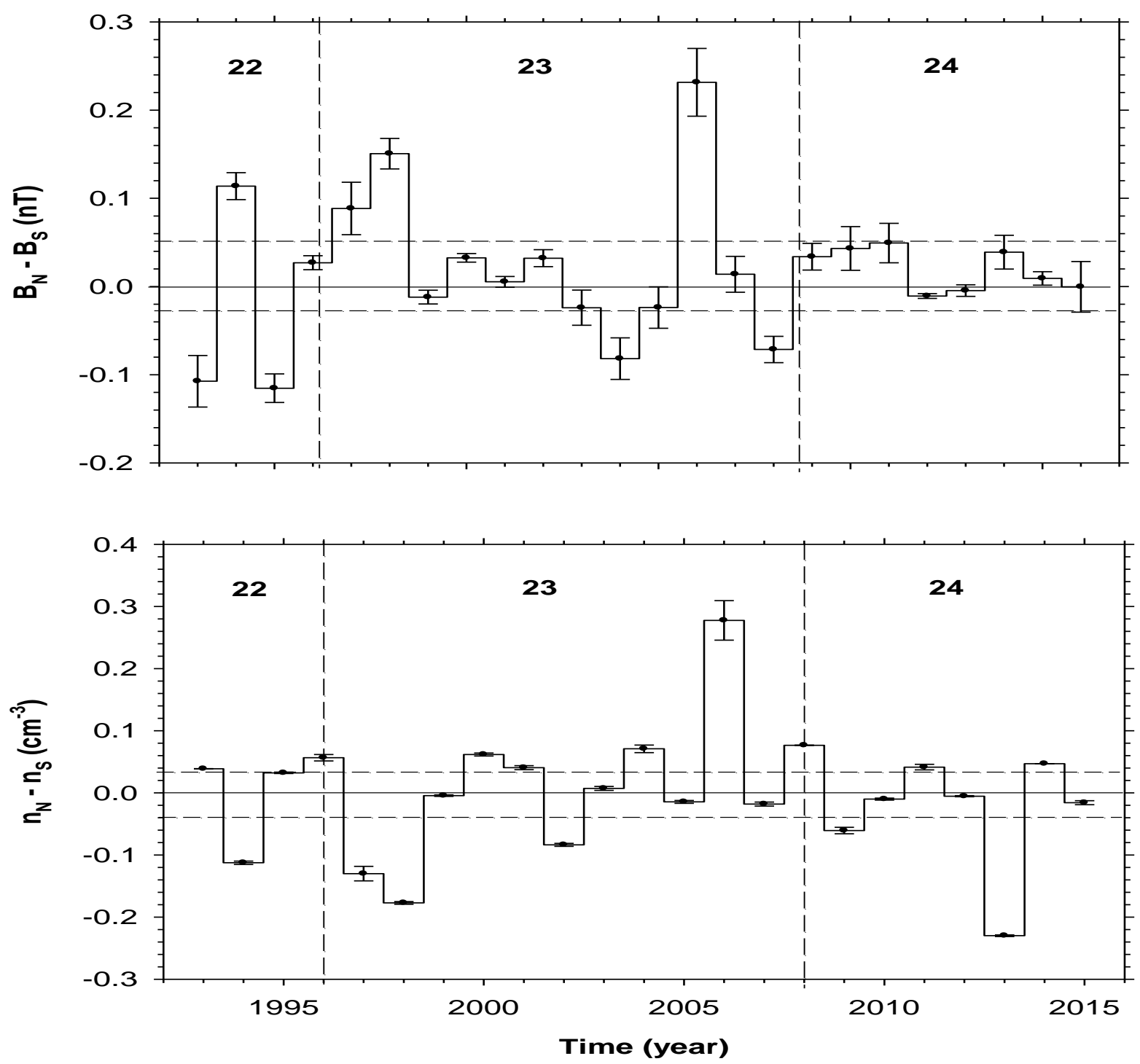

Fig. (4): The 3-yr running averages of N-S asymmetries for IMF magnitude (plot a) and for plasma density (plot b) over the period (1992-2017) based on the hemispheric distribution of SSNs. The vertical dashed lines denote the minima of solar activity. The horizontal dashed lines represent the $95 \%$ confidence levels for positive and negative values of $\mathrm{N}-\mathrm{S}$ asymmetry. The solar cycles are numbered on each plot. 
In the bottom plot of Figure (4), when we take the error bars as well as the confidence level in our consideration, there are 14 years out of 23 with $\mathrm{N}-\mathrm{S}$ asymmetry in the yearly average of plasma density $n$. Eight clear positive asymmetries have plasma density for the northern sorted group greater than the southern one, happened in 1996, 2000, 2001, 2004, 2006, 2008, 2011, and 2014. On the other hand, six clear negative N-S asymmetries occurred in 1994, 1997, 1998, 2002, 2009, and 2013. The largest positive asymmetry $\left(0.278 \pm 0.032 \mathrm{~cm}^{-3}\right)$ occurs in 2006 through the solar cycle 23 . In contrast, the most significant negative asymmetry $\left(-0.230 \pm 0.00123 \mathrm{~cm}^{-3}\right)$ occurs in 2013 through the solar cycle 24.

Figure (5) (top plot) shows the yearly differences between northern and southern sorted groups for solar plasma velocity $\left(\mathrm{V}_{\mathrm{N}}-\mathrm{V}_{\mathrm{S}}\right)$. The solar plasma of northern sorted group is faster than that of southern sorted group during years; 1994, 2000, 2006, and 2008. In contrast, the northern sorted group is slower than that of southern sorted one in years 1993, 1996, 2002, 2011, and 2013. In addition, the solar plasma for the northern sorted group is faster by $11.07 \pm 1.77 \mathrm{~km} / \mathrm{s}$ than that for the southern sorted group in 2000 (cycle 23), whereas the solar plasma for the northern sorted group was slower by $9.23 \pm 0.84 \mathrm{~km} / \mathrm{s}$ than that for the southern sorted group in 2013 (cycle 24).
Figure (5) (bottom plot) represents the N-S asymmetry of yearly average for the plasma temperature $(T)$. The plot displays eleven years with significant asymmetry between the northern and southern groups for the solar plasma temperature. The years; 1994, 1995, 2000, 2006 and 2008, reveal that the solar plasma is hotter for the northern sorted group than that of the southern one. In contrast, the years; 1993, 1996, 2002, 2004, 2011 and 2013, exhibit the reverse. Furthermore, the solar plasma for the northern sorted group is hotter by $(6.67 \pm 1.18) \times 10^{3} \mathrm{~K}$ than that for the southern sorted one in 2008. On the other hand, solar plasma temperature for the southern sorted group predominated the northern one by $(4.83 \pm 0.43) \times 10^{3} \mathrm{~K}$ in 2013 .

The time variation of the north-south asymmetry for the plasma pressure $\left[\mathrm{P}_{\mathrm{N}}-\mathrm{P}_{\mathrm{S}}\right]$ is shown in Figure (6). The northern dominance is indicated in the years: 1994, 1996, 2000, 2006 and 2008. While the years: 1993, 2004, and 2011-2014 manifest a southern dominance. The higher significant northern asymmetry $(0.083 \pm 0.012 \mathrm{nPa})$ occurs in 2000 (during the ascending of the solar cycle 23) and the peak; $(-0.077 \pm 0.007 \mathrm{nPa})$ appearing in 2013 (near to the maximum of solar cycle 24 is the greatest southern dominance peak.
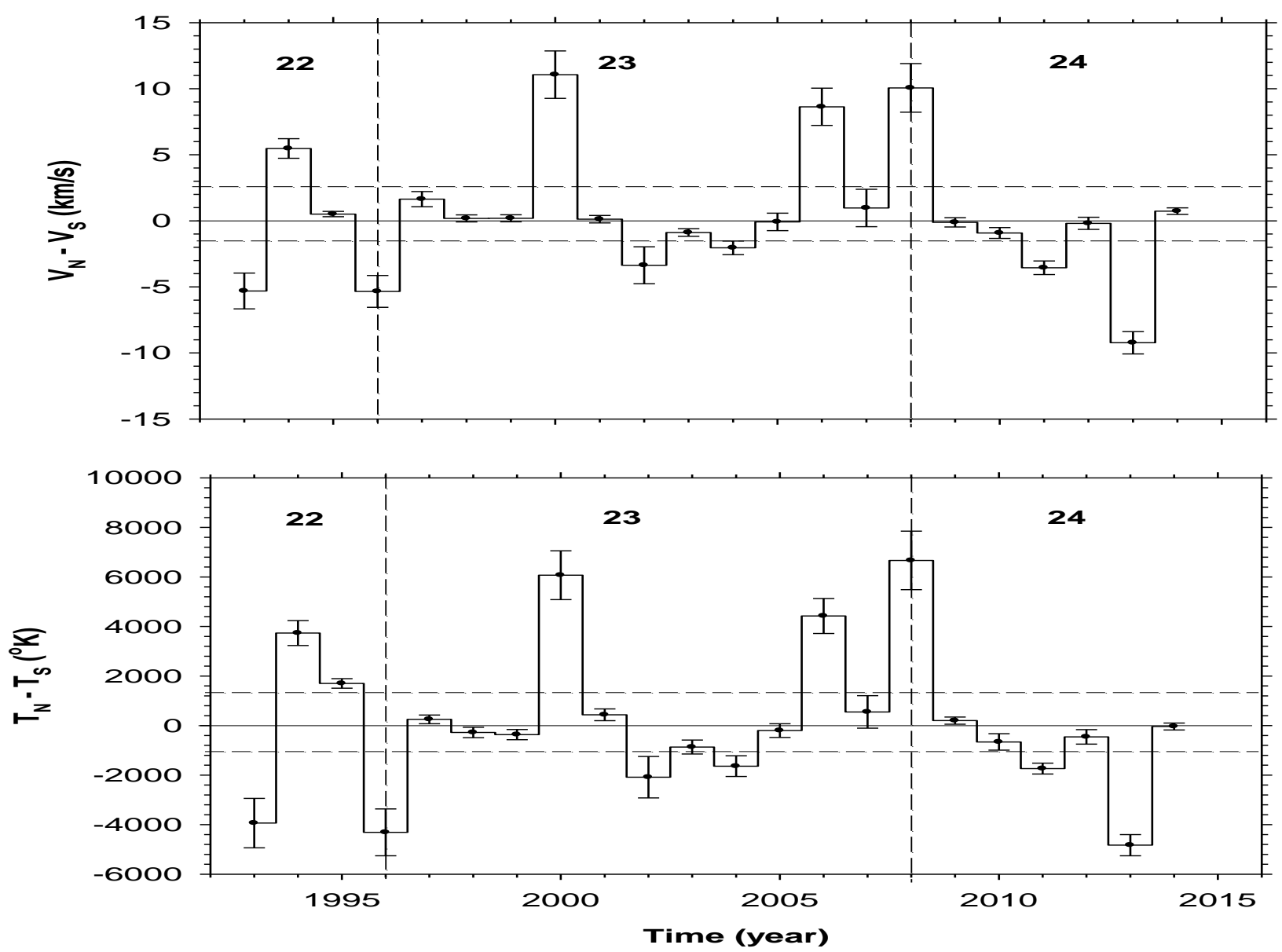

Fig. (5): The 3-yr running averages of N-S asymmetries for solar plasma velocity (plot a) and for plasma temperature (plot b) over the period (1992-2017). The notations are the same as in Fig. 4 


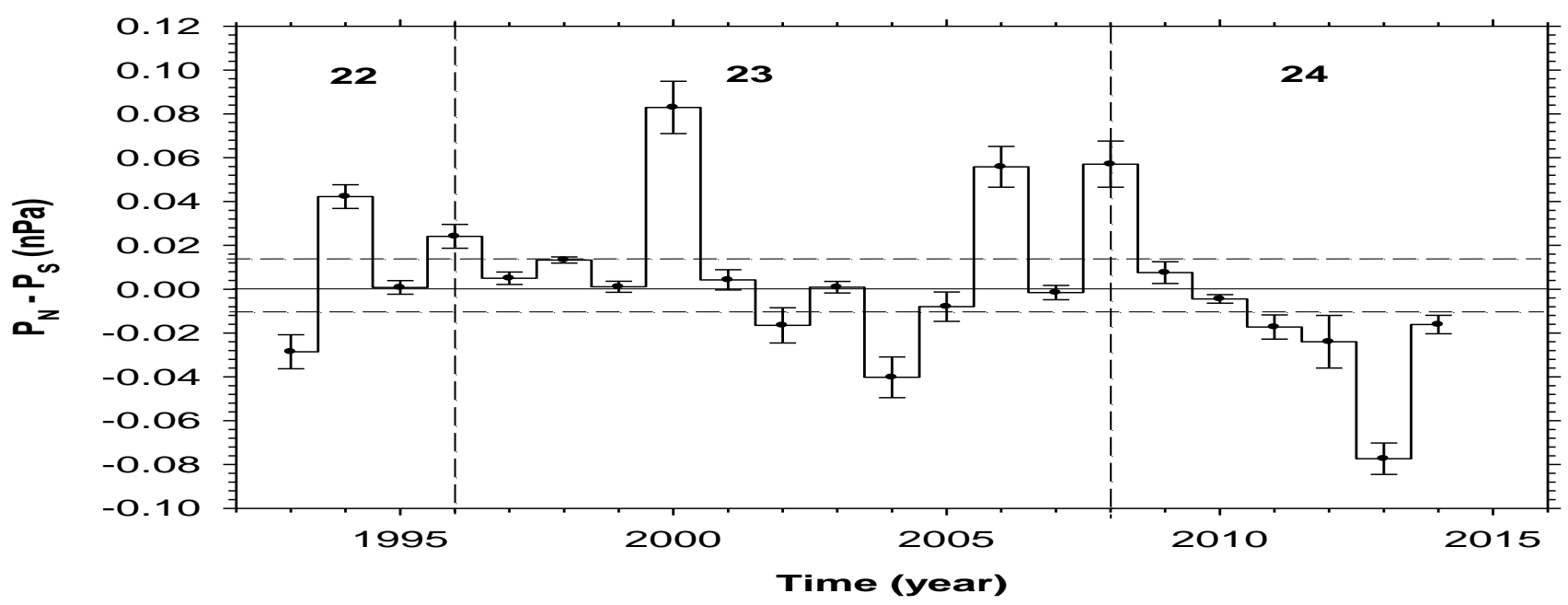

Fig. (6): The 3-yr running averages of N-S asymmetries for solar plasma pressure (plot a) over the period (1992-2017). The notations are the same as in Fig. (4)

Tables (6-8) list the grand averages of SSNs, $B, n, T$, $V$, and $P$ for northern and southern hemisphere over the solar cycles 22, 23 and 24, Furthermore numbers of days and their percentage according to the total number of days during each cycle are calculated. The solar cycles 22 and 23 exhibit southern dominance of $S S N s$, while the solar cycle 24 have a northern dominance. In addition to the asymmetry between the northern and southern sorted groups for $n, P$ and $B$ are not detected significantly for the solar cycles $22-24$. The plasma temperature and solar wind speed parameters have southern dominance for the solar cycles 22 and 24 while the cycle 23 shows more northern than southern. The solar plasma for southern group is hotter by $7.2 \times 10^{3} \mathrm{~K}, 11 \times 10^{3} \mathrm{~K}$ during cycle 22 and 24 than that in the northern group while the cycle 23 reveals the plasma particles hotter by $9 \times 10^{3} \mathrm{~K}$ in the northern than that in southern. The plasma particles are faster in southern hemisphere by $22 \mathrm{~km} / \mathrm{s}$ and $21 \mathrm{~km} / \mathrm{s}$ during the solar cycles 22 and 24 , respectively than that in the northern while for the solar cycle 23 , the plasma particles for northern group is faster by $5 \mathrm{~km} / \mathrm{s}$ than that in the southern.

The results of this section, obtained following the dominance of the hemispheric SSNs, reveal a good agreement with those of section 3.1, yielded when considering the preference of the hemispheric SSAs, through the overlapped period of 1992-2017.

Table (6): Grand averages of IMF magnitude, solar and plasma parameters during solar activity cycle 22 based on the hemispheric SSNs. The number of the northern and the southern days and their percentages are also listed.

\begin{tabular}{cccc}
\hline $\begin{array}{l}\text { IMF magnitude, } \\
\text { solar, and plasma } \\
\text { parameters }\end{array}$ & $\begin{array}{c}\text { No. northern days } \\
\mathbf{6 7 9}\end{array}$ & $\begin{array}{c}(\%) \\
\mathbf{4 0 . 6}\end{array}$ & \multicolumn{2}{c}{ No. southern days (\%) } \\
$\mathbf{8 0 4}$ & $\mathbf{4 8}$ & \\
\hline & Average & Average & $(\mathrm{N}-\mathrm{S})$ \\
$\mathrm{SSAs}(\mu \mathrm{Hemi})$ & $27.5 \pm 0.69$ & $34.8 \pm 0.96$ & -7.3 \\
$\mathrm{~B}(\mathrm{nT})$ & $6.13 \pm 0.11$ & $6.83 \pm 0.13$ & -0.70 \\
$\mathrm{n}\left(\mathrm{cm}^{-3}\right)$ & $8.30 \pm 0.21$ & $8.09 \pm 0.19$ & 0.21 \\
$\mathrm{~T} \times 10^{3}\left({ }^{\mathrm{o}} \mathrm{K}\right)$ & $93 \pm 2.77$ & $100.2 \pm 3.08$ & -7.2 \\
$\mathrm{~V}(\mathrm{~km} / \mathrm{s})$ & $425 \pm 3.63$ & $447 \pm 4.18$ & -22 \\
$\mathrm{P}(\mathrm{nPa})$ & $2.49 \pm 0.04$ & $2.52 \pm 0.05$ & -0.03 \\
\hline
\end{tabular}

Table (7): Grand averages of IMF magnitude, solar and plasma parameters during solar activity cycle 23 based on the hemispheric SSNs. The number of the northern and the southern days and their percentages are also listed.

\begin{tabular}{ccccc}
\hline $\begin{array}{l}\text { IMF magnitude, } \\
\text { solar, and plasma } \\
\text { parameters }\end{array}$ & $\begin{array}{l}\text { No. northern days } \\
\mathbf{1 5 9 1}\end{array}$ & $\begin{array}{c}\text { (\%) } \\
\mathbf{3 8 . 2}\end{array}$ & \multicolumn{2}{c}{ No. southern days (\%) } \\
$\mathbf{2 1 5 7}$ & $\mathbf{5 1 . 7}$ & \\
\hline & Average & Average & $(\mathrm{N}-\mathrm{S})$ \\
$\mathrm{SSAs}(\mu \mathrm{Hemi})$ & $41.7 \pm 0.675$ & $47.2 \pm 0.62$ & -5.5 \\
$\mathrm{~B}(\mathrm{nT})$ & $6.80 \pm 0.073$ & $6.36 \pm 0.059$ & 0.44 \\
$\mathrm{n}\left(\mathrm{cm}^{-3}\right)$ & $6.20 \pm 0.103$ & $6.04 \pm 0.084$ & 0.16 \\
$\mathrm{~T} \times 10^{3}\left({ }^{\mathrm{o}} \mathrm{K}\right)$ & $111 \pm 2.03$ & $102 \pm 1.72$ & 9 \\
$\mathrm{~V}(\mathrm{~km} / \mathrm{s})$ & $452 \pm 2.57$ & $447 \pm 2.320$ & 5 \\
$\mathrm{P}(\mathrm{nPa})$ & $2.17 \pm 0.04$ & $2.07 \pm 0.03$ & 0.10 \\
\hline
\end{tabular}

Table (8): Grand averages of IMF magnitude, solar and plasma parameters during solar activity cycle 24 based on the hemispheric SSNs. The number of the northern and the southern days and their percentages are also listed.

\begin{tabular}{cccc}
\hline $\begin{array}{l}\text { IMF magnitude, } \\
\text { solar, and plasma } \\
\text { parameters }\end{array}$ & $\begin{array}{c}\text { No. northern days } \\
\mathbf{1 6 9 9}\end{array}$ & $\begin{array}{c}(\%) \\
\mathbf{4 6 . 5}\end{array}$ & \multicolumn{3}{c}{ No. southern days (\%) } \\
\hline & Average & Average & $(\mathrm{N}-\mathrm{S})$ \\
$\mathrm{SSAs}(\mu \mathrm{Hemi})$ & $27.8 \pm 0.45$ & $26 \pm 0.53$ & 1.8 \\
$\mathrm{~B}(\mathrm{nT})$ & $5.47 \pm 0.054$ & $5.64 \pm 0.068$ & -0.17 \\
$\mathrm{n}\left(\mathrm{cm}^{-3}\right)$ & $6.00 \pm 0.10$ & $6.40 \pm 0.12$ & -0.40 \\
$\mathrm{~T} \times 10^{3}\left({ }^{\mathrm{o}} \mathrm{K}\right)$ & $84 \pm 1.93$ & $95 \pm 1.99$ & -11 \\
$\mathrm{~V}(\mathrm{~km} / \mathrm{s})$ & $412 \pm 2.54$ & $433 \pm 2.80$ & -21 \\
$\mathrm{P}(\mathrm{nPa})$ & $1.93 \pm 0.033$ & $2.13 \pm 0.035$ & -0.200 \\
\hline
\end{tabular}

\section{SUMMARY AND CONCLUSIONS}

Daily data of SSNS and SSAs as well as the interplanetary plasma parameters $(B, n, V, T$, and $P$ ) during the period 1967-2017 have been examined in order to investigate the influence of the solar activity distributions on the northern and the southern solar hemispheres (the dominance of either northern or southern solar activities) on the interplanetary plasma parameters. A consistent long-term variations between 
SSNs and each interplanetary plasma parameter has been observed only when the daily data has been smoothed over 3-yr as well as a specific time-lag (2-yr for $V, T$, and $P$ as well as 1-yr for $n$ ) has been applied. In addition, the solar activity and the interplanetary plasma parameters showed a good correlation if the running means over 3-yr and a specific time lags are taken into our consideration. After applying the previous analyses, the daily data of each interplanetary plasma parameter has been classified into two groups, northern $(N)$ and southern $(S)$ then the yearly averages of the sorted $N$ and $S$ groups have been calculated for each parameter. The absolute asymmetry index $(N-S)$ between the northern and southern groups, for each considered parameter, has been presented. Thus, if $(N-S)>0$, the parameter value for the sorted northern group predominates that for the southern sorted group, whereas if $(N-S)<0$, the reverse is true. The interplanetary plasma parameters showed asymmetry behavior between the northern and the southern sorted groups during random years of the entire period. Our conclusions are summarized as the following:

1- The asymmetry between the northern and southern sorted groups for the interplanetary magnitude $B$ showed a dominance of 13 years for the northern sorted group which occurred in solar cycles 20-24. In contrast, 12 years for the southern sorted group throughout the cycles 20-23. The most prominent peak of the northern sorted group located at 2006 $(0.25 \pm 0.042 \mathrm{nT})$ during the descending phase of cycle 23 . On the other hand, the most significant ones for the southern sorted group occurred in 1978 $(0.15 \pm 0.027 \mathrm{nT})$ and $1987(0.134 \pm 0.027 \mathrm{nT})$ during the ascending phases of cycles 21 and 22, respectively. Additionally, the asymmetry between the northern and the southern groups for the grand average of $B$ over the solar activity cycles is not detectable, indicating that the solar cycles are magnetically symmetric

2- Within the limit of accuracy (95\% confidence levels), during random 13 years, the solar plasma was denser for the northern sorted group than that of the southern sorted one, whereas 17 different random years showed the reverse. The most significant peaks which have positive asymmetry index are found in $1970\left(0.306 \pm 0.029 \mathrm{~cm}^{-3}\right), 1974\left(0.231 \pm 0.031 \mathrm{~cm}^{-3}\right)$, and $2006\left(0.303 \pm 0.055 \mathrm{~cm}^{-3}\right)$. On the other hand, the most prominent peak for the southern sorted group is located in $2013\left(0.230 \pm 0.031 \mathrm{~cm}^{-3}\right)$. Also, the asymmetry between the northern and the southern groups for the solar plasma density over the solar activity cycle is statistically insignificant.
3- The solar plasma was faster for the northern sorted group than that of southern sorted one through six years (1970, 1986, 1994, 2000, 2006 and 2008) and in contrast, different ten years $(1971,1974,1977$, 1978, 1983, 1985, 1993 and 1996, 2011, and 2013) showed the reverse. In addition, the solar plasma for the northern sorted group was faster by $12.4 \pm 1.3$ $\mathrm{km} / \mathrm{s}$ and $12.2 \pm 1.8 \mathrm{~km} / \mathrm{s}$ than that for the southern sorted group in 1970 and 2000, respectively. Regarding the southern sorted group, the solar plasma was faster by $7 \pm 1.8 \mathrm{~km} / \mathrm{s}$ and $79 \pm 0.86 \mathrm{~km} / \mathrm{s}$ than that for the northern sorted group during 1971 and 2013.

4- The asymmetry between the northern and the southern sorted groups for plasma temperature exhibits coupling at some level with the plasma velocity (both plots nearly symmetric). Regarding the northern sorted group, the highest temperatures of the solar plasma were $9088 \pm 948.7{ }^{\circ} \mathrm{K}$ (in 1970 during the ascending phase of cycle 20), $6671 \pm 967.5^{\circ} \mathrm{K}$ (in 2000 ), and $7100 \pm 1232{ }^{\circ} \mathrm{K}$ (in 2008 at minimum of solar activity). In contrast, for the southern sorted group, the highest temperatures of the solar plasma were $5270 \pm 1306{ }^{\circ} \mathrm{K}$ (in 1971, during the ascending phase of cycle 20) and $4636 \pm 436.3{ }^{\circ} \mathrm{K}$ (in 2013 , near to maximum activity of solar cycle 24). Furthermore, the solar plasma for the southern sorted group was hotter by $15.7 \times 10^{3}{ }^{\circ} \mathrm{K}, 8 \times 10^{3}{ }^{\circ} \mathrm{K}$, and $14.5 \times 10^{3}{ }^{\circ} \mathrm{K}$ than that for the northern sorted one during the solar cycles 20,21, and 24, respectively. In contrast, during the solar cycle 23 , the solar plasma for the southern sorted group was colder by $7.2 \times 10^{3}{ }^{\circ} \mathrm{K}$ than that for the northern sorted group. Also, the solar plasma for the southern sorted group was faster by $20.3 \mathrm{~km} / \mathrm{s}, 13 \mathrm{~km} / \mathrm{s}, 5 \mathrm{~km} / \mathrm{s}$, and $29 \mathrm{~km} / \mathrm{s}$ than that for the northern sorted one during the solar cycles 20,21, 22 , and 24 , respectively. In contrast, during the solar cycle 23 , the solar plasma for the southern sorted group was slower by $7.4 \mathrm{~km} / \mathrm{s}$ than that for northern sorted group.

5- The asymmetry of plasma dynamic pressure $(P)$ between the northern and the southern sorted groups showed ten years $(1970,1980,1986,1989,1991$, 1994, 1996, 2000, 2006, and 2008) have the dominance of the northern sorted group for the plasma dynamic pressure over the southern sorted one. On the other hand, 12 years $(1968,1971,1976$, 1977, 1978, 1983, 1987, 1993, 2004, 2011, 2013, and 2014) have plasma dynamic pressure for the southern sorted group greater than that of the northern group. The most significant northern and southern peaks are 
$0.117 \pm 0.012 \mathrm{nPa}$ and $0.075 \pm 0.0041 \mathrm{nPa}$ occurred in 1970 and 2013, respectively.

6-The asymmetry of IMF magnitude $(B)$ based on SSNS shows four years have northern dominance through (1994, 1997, 1998, and 2006). Also, four years (1993, 1995, 2004, and 2008) showed southern dominance.

7-The asymmetry of between the northern and southern sorted groups based on the distribution of hemispheric SSNs for ion plasma density ( $n$ ) shows 14 years exhibit clear N-S asymmetry. Eight clear positive asymmetries have plasma density for the northern sorted group greater than the southern one. In contrast, six clear negative N-S asymmetries. The largest positive asymmetry $\left(0.278 \pm 0.032 \mathrm{~cm}^{-3}\right)$ occurs in 2006 through the solar cycle 23. In contrast, the most significant negative asymmetry ($0.230 \pm 0.00123 \mathrm{~cm}^{-3}$ ) occurs in 2013 through the solar cycle 24.

8-The asymmetry between the northern and the southern sorted groups for plasma pressure $(P)$ and plasma temperature $(T)$ reveals five years have a dominance of northern sorted group and six years have southern dominance.

9- Finally, a study of the asymmetry between the northern and the southern sorted groups of the interplanetary parameters on the basis on the hemispheric SSNS revealed a good matching with the asymmetry based on the hemispheric SSAs most of the years during the common period (1992-2017).

\section{ACKNOWLEDGEMENTS}

We wish to thank the anonymous referees for their careful reading of our manuscript and for constructive comments and suggestions that improved the original version. The authors are thankful to the OMNI database from the National Space Science Data Centre (http://www.omniweb.gsfc.nasa.gov).

\section{REFERENCES}

[1] M. A. El-Borie, A. M. El-Taher, N. E. Aly, and A. A. Bishara, Astroparticle Physics, 100, 13-21, (2018).

[2] D. H. Hathaway, Living Reviews in Solar Physics, 12 (1), 4, (2015).
[3] K. J. Li, J. Qiu, T. W. Su, and P. X. Gao, The Astrophysical Journal, 621 (1), L81-L84, (2005).

[4] M. Carbonell, R. Oliver, and J. L. Ballester, Astronomy and Astrophysics, 274, 497, (1993).

[5] G. VIZOSO and J. BALLESTER, Astronomy and astrophysics (Berlin. Print), 229 (2), 540 546, (1990).

[6] D. B. Swinson, M. A. Shea, and J. E. Humble, Journal of Geophysical Research, 91 (A3), 2943, (1986).

[7] P. J. Pulkkinen, J. Brooke, J. Pelt, and I. Tbominen, Astronomy and Astrophysics, 341 (2). 1999.

[8] D. Swinson and S. Yasue, (1991).

[9] M. A. El-Borie, Astroparticle Physics, 10 (2-3), 165-174, (1999).

[10] M. A. El-Borie, IL NUOVO CIMENTO, 24 C (6), (2001).

[11] B. Zieger and K. Mursula, Geophysical Research Letters, 25 (6), 841-844, (1998).

[12] N. U. Crooker et al., Journal of Geophysical Research A: Space Physics, 102 (A3), 46734679, (1997).

[13] M. A. El-Borie, A. A.-M. Abdel-Halim, and S. Y. El-Monier, International Journal of Astronomy and Astrophysics, 06 (01), 14-22, (2016).

[14] M. A. El-Borie, A. A. Abdel-Halim, S. Y. ElMonier, and A. A. Bishara, in Journal of Physics: Conference Series, 2017, 869 (1).

[15] G. Makarov, Solnechno-Zemnaya Fizika, 2 (1), 32-35, (2016).

[16] M. A. El-Borie, A. M. El-Taher, N. E. Aly, and A. A. Bishara, Physics of Plasmas, 25 (4), 042901, (2018).

[17] M. A. El-Borie, A. M. El-Taher, A. A. Thabet, and A. A. Bishara, Advances in Space Research, 64 (1), 278-286, (2019).

[18] W. Köhnlein, Astrophysics and Space Science, 245 (1), 81-88, (1996). 